\title{
Uma Fisiologia da Paisagem: Locomoção, GIS e Sites Catchment. Uma Nova Perspectiva.
}

\author{
José Roberto Pellini*
}

\begin{abstract}
PELLINI, J.R. Uma Fisiologia da Paisagem: Locomoção, GIS e Sites Catchment. Uma Nova Perspectiva. Revista do Museu de Arqueologia e Etnologia, São Paulo, 17: 23-37, 2007.
\end{abstract}

Resumo: Locomoção e distância são elementos essenciais da vida humana. Mas a despeito de sua importância estes conceitos têm sido tratados de maneira simplista pela arqueologia, principalmente no que se refere às Analises de Site Catchment. Os modelos mais tradicionais de Site Catchment tratam o espaço como um elemento cartesiano neutro dissociado de seu conteúdo social. Nos últimos anos o uso do GIS tem permitido a determinação de Site Catchments de maneira mais realista, principalmente ao considerar fatores fisiológicos e elementos de percepção na modelagem dos territórios de exploração. A proposta deste artigo é apresentar um exemplo do uso destes novos modelos e testar sua validade como método de interpretação.

Palavras-chave: Site Catchment - Locomoção - GIS.

\section{Introdução}

E nquanto a chamada arqueologia da abrangência e a complexidade dos processos que ocorrem dentro da paisagem através da incorporação de elementos originados em campos como história social, sociologia e fenomenologia, estas incorporações não têm tido seu reflexo no desenvolvimento de novas metodologias de análise. Segundo Llobera (2000), indubitavel-mente as abordagens da arqueologia da paisagem incorporam importantes avanços teóricos que têm deixado os arqueólogos sensíveis aos

$\left.{ }^{*}\right)$ Museu de Arqueologia e Etnologia. Pós-doutorado. jrpellini@yahoo.com.br intrincados elementos do espaço, mas estes avanços não estão livres de deficiências. A principal delas é a pouca importância que se tem dado a processos de percepção, movimento e interação.

Movimento é um aspecto central na história humana. É através do movimento que os indivíduos concebem e alteram sua percepção do meio. É através do movimento que os indivíduos criam um senso de espacialidade que não ocorre em um espaço cartesiano vazio. O meio existe somente em relação aos observadores e se pensarmos que estes observadores são móveis, sua percepção e seu conhecimento sobre o meio são experimentados através do movimento. Movimento e locomoção determinam a acessibilidade a recursos, produtos e serviços. Mobilidade estabelece interação com locais que apresentam oportunidades e 
vantagens econômicas ou socioculturais. Mas a despeito de sua importância, movimento tem sido tratado de forma simplista pela arqueologia. Parte do problema não está associada à definição de movimento em si, mas de espaço e distância.

Os modelos tradicionais de site catchment, por exemplo, tentam impor uma geometria cartesiana, abstrata e atemporal sobre uma realidade que é fundamentalmente reflexiva, subjetiva e contingente. $\mathrm{O}$ espaço nesses casos é visto como uma entidade puramente física, separada do tempo e expressa como um valor absoluto. Sabemos que o espaço não é na realidade um conceito cartesiano neutro, mas é socialmente construído.

A idéia central das análises de site catchment propostas por Vita-Finzi e Higgs (1970) é que o custo-benefício do investimento energético e do retorno econômico decai exponencialmente conforme aumenta a distância em relação ao centro do assentamento. Quando o limite econômico ou energético é alcançado, o custobenefício de exploração do território se torna negativo. A premissa básica é que quanto mais longe do sítio as fontes de recursos estão, maiores são os custos econômicos ou energéticos para explorá-los. Normalmente há um ponto em que o benefício econômico se iguala ao custo de exploração. Sendo assim, um limite econômico ou energético pode ser definido neste ponto para determinar o território de exploração do sítio. Como ressaltou Gaffney e Stancic (1991), estudos etnográficos demonstraram que este território limite varia entre sociedades mais móveis, como a dos caçadorescoletores, e entre sociedades mais sedentárias, como as dos horticultores. Limites de 10 e 5 $\mathrm{km}$ são sugeridos para estas sociedades respectivamente

Tradicionalmente, o primeiro passo nas análises de site catchment é a determinação de um território (catchment) associado a um dado ponto focal (sítio), através da utilização de algum pressuposto geográfico. Os dois modelos mais utilizados para a determinação de um território de exploração são os modelos que se baseiam na identificação de limites naturais ou em distância.
No primeiro modelo, as zonas territoriais são baseadas em regiões naturais como corpos de água, vales e montanhas. Como os catchments representam delimitações de caráter social, político e econômico, tal modelo falha na tentativa de recriar os territórios de exploração, pois leva em conta apenas a fisiografia local, deixando de fora aspectos culturais e mesmo fisiológicos.

Já nos modelos que utilizam distância como meio de determinar a forma e a extensão dos catchments, um raio predefinido de distância é determinado ao redor do sítio. $\mathrm{O}$ resultado assim seria um catchment definido a partir de um raio variante em quilômetros ou metros ao redor do sítio. $\mathrm{O}$ raio de distância poderia ser escolhido a partir de observações empíricas ou baseado em dados etnográficos. O grande problema relacionado a este tipo de metodologia é a maneira simplista na qual a paisagem é retratada. Na paisagem real, o uso e o controle socioeconômico de um dado território não terminam abruptamente conforme se cruza o limite de um território. Em vez disso ele torna-se menor conforme aumenta a distância para o centro do território. Empregar um raio de distância fixo para definir um catchment é o equivalente a se deslocar em uma superfície plana e regular, onde o custo associado à locomoção é o mesmo em todas as direções, ou seja, isotrópico. ${ }^{1}$ Neste contexto, o catchment é definido apenas pela máxima distância horizontal a partir do centro do assentamento. Distância aqui é vista em termos euclidianos, não levando em conta o contexto sociocultural dos sistemas analisados. $\mathrm{O}$ espaço é visto como um produto cartesiano que é abstraído de seu contexto. Como apontou Llobera (1996), não há observadores, não há perspectiva, não há história.

Segundo Morril e Pitts (1967), se nós analisarmos a locomoção de certo indivíduo, podemos determinar de antemão que somente

(1) Análises isotrópicas de custo são utilizadas onde o custo de retorno à base não está sendo calculado ou em casos em que a direção do movimento através de uma dada superfície de fricção não afeta o custo da movimentação. 
uma viagem é possível em um momento específico de tempo. Neste momento o indivíduo é confrontado com um grande número de possíveis destinos, direções e distância, mas evidentemente apenas uma escolha pode ser feita. Mesmo que seu movimento não possa ser determinado com precisão, sabemos que o destino escolhido é influenciado por forças sistemáticas. Para os autores, os movimentos de um indivíduo são governados por uma relação entre informação e distância. A informação consiste no conhecimento que um indivíduo tem do seu mundo, e seu campo de informação é a distribuição espacial deste conhecimento.

Como sugeriu Hagerstrand (1962), a probabilidade de um indivíduo se mover de um local para o outro precisa ser computada como uma função das características do indivíduo (idade, raça, sexo, vida urbana ou rural etc.), das características da área estudada (formas de relevo, paisagens, formações urbanas, contexto socioeconômico), das características dos possíveis destinos, bem como das diferenças entre eles.

Embora muitos arqueólogos venham utilizando distância linear reta entre pontos de interesse como fator de análise dos padrões de locomoção e para a determinação do site catchment, sabemos que no mundo real a localização de fontes de recursos ou a área de exploração territorial não é limitada pela distância linear direta para algum lugar. Pelo contrário, é o custo de alcançar um dado ponto na paisagem que condiciona o movimento e a locomoção, ou seja, o custo de se alcançar um dado ponto ou recurso é uma função do esforço requerido para cruzar as distâncias entre os dois pontos. Para Whitley and Hicks (2003), a localização de assentamentos ou a exploração de territórios não é limitada pela distância linear para algum ponto específico, isto porque o custo de alcançar um dado local ou recurso é uma função do esforço requerido para se transpor a distância entre o ponto inicial e o ponto final. Este esforço é chamado custo de distância. Determinar um site catchment a partir dos custos envolvidos nos processos de locomoção e deslocamento representa um meio mais realístico de definir o território associado a um dado assentamento.

\section{Índices fisiológicos de deslocamento e locomoção}

A grande maioria dos softwares de GIS oferece a possibilidade de gerar superfícies de custo. Estas superfícies de custo podem ser consideradas como um produto baseado em proximidades contínuas que levam em conta não somente a proximidade, como também o caráter do terreno sobre o qual as proximidades são calculadas. Superfície de custo, assim como distância, é um modelo matemático cujo significado arqueológico não é fixado, cabendo ao arqueólogo definir os parâmetros de análise.

Uma superfície de custo é um modelo computadorizado da paisagem no qual para cada parte da superfície é assinalado um valor, ou custo, que representa o esforço ou energia requerida para alcançar certo ponto a partir de um ponto predeterminado. $O$ custo total é determinado por um logaritmo que incorpora não só as distâncias em relação ao ponto final, mas também os custos adicionais relacionados a aspectos particulares da paisagem. Estes custos adicionais são determinados pelo pesquisador e são derivados de sistemas de classificação nos quais para cada característica da paisagem é determinado certo valor de custo.

A chave para um modelo de custo que realmente represente o custo envolvido no deslocamento dos indivíduos dentro da paisagem é a escolha dos valores de fricção mais apropriados.

O custo de locomoção tem sido alvo freqüente em trabalhos de arqueologia (Duggan e Haisman 1992; Limp 1990; Beck e Taylor 2003; Balstrom 2002). Nestes trabalhos, a forma mais simples de superfície de fricção é a declividade do terreno. Em teoria, o custo de deslocamento associado a uma parcela do terreno aumenta conforme aumenta a declividade. 
O ponto a ser considerado quando utilizamos modelos de inclinação para a criação de superfícies de custo é que a inclinação não exerce a mesma força uniformemente em todas as direções. Isto porque quando o movimento é perpendicular à inclinação o esforço exercido é menor. Neste sentido, o uso de modelos de custo de superfície anisotrópicos é mais eficiente, pois leva em conta tanto a magnitude quanto a direção do movimento entre o ponto $\mathrm{A}$ e $\mathrm{B}$, o que produz uma superfície de custo direcional na qual a direção é calculada contra a suavidade e o aspecto da inclinação.

A fim de contornar as deficiências dos modelos de catchment que utilizam declividade como aspecto de determinação de custo, modelos mais recentes têm utilizado tempo, e não distância, como um fator determinante na criação do catchment. A principal inovação é o uso de medidas fisiológicas de gasto de energia para representar o custo de deslocamento de maneira mais realística.

A idéia central destes modelos é transformar os fatores de declividade e elevação em índices que mostrem gasto calórico, velocidade ou tempo de deslocamento. Verhagen et al. (1999) utilizou, por exemplo, a função desenvolvida por Imholf (1950) e reformulada pelo geógrafo Waldo Tobler (1993), em que o efeito da declividade sobre a velocidade do deslocamento é expressa por:

$\mathrm{V}=6 \exp (-3,5(\mathrm{~S}+0,05))$

onde (V) representa a velocidade em $\mathrm{km} / \mathrm{h},(\mathrm{S})$ é a declividade do terreno expressa em porcentagem e (Exp) é a base natural de logaritmo. Neste caso, a declividade do terreno descrita em porcentagem é modificada para representar a velocidade de caminhamento de acordo com as diferenças e especificidades do terreno.

Desta maneira, o resultado final irá representar uma superfície que expressa a velocidade que um ser humano pode atingir de acordo com o tipo de terreno. Com base nas velocidades praticadas é possível definir um catchment a partir do centro do sítio tomando por base os padrões de mobilidade descritos por Vita-Finzi e Higgs (1970), que pressupõem um catchment de $10 \mathrm{~km}$ em média para sociedades de caçadores-coletores e $5 \mathrm{~km}$ para sociedades horticultoras. O método de análise é orientado, o que significa dizer que se a resolução da célula de elevação for de 30 x 30 m, o resultado irá expressar a velocidade necessária para se deslocar $30 \mathrm{~m}$ dentro da paisagem de acordo com o tipo de terreno.

Um modelo de deslocamento um pouco diferente foi utilizado por Marbe (1996), que utilizou a fórmula desenvolvida por Pandolf (1977), que calcula o gasto fisiológico em watts a partir das diferenças de declividade do terreno para determinar o limite do catchment. A diferença da fórmula utilizada por Marbe com a fórmula de Tobler é que a fórmula de Pandolf leva em conta as características do indivíduo nas análises. A fórmula é descrita como:

$\mathrm{M}=1,5+2(\mathrm{~W}+\mathrm{L})(\mathrm{L} / \mathrm{W})^{2}+\mathrm{N}(\mathrm{W}+\mathrm{L})\left(1,5 \mathrm{~V}^{2}+\right.$ $0.35 \mathrm{VG})$

onde (M) é a taxa metabólica expressa em watts, (W) é o peso corporal, (L) é o peso da carga extra, $(V)$ velocidade, $(G)$ é a declividade do terreno. Leusen modificou a fórmula de Pandolf alterando o valor final de $(G)$ para representar a simetria da declividade como expressa por Llobera (2000), substituindo-o pelo valor absoluto $(\mathrm{G}+6)$. $\mathrm{O}$ resultado esperado é uma superfície que apresenta os valores calóricos em watts a partir do centro do sítio. O limite do catchment neste caso seria o chamado optimum energético, ou seja, a distância na qual o gasto calórico se iguala ao ganho calórico.

Outra variação da fórmula de Pandolf foi sugerida por Wood e Wood (2006). Segundo os autores, a fórmula inicial não considerava a direção do movimento como fator de fricção. Sendo assim, seria necessário utilizar a fórmula original para declividades positivas e planas e uma derivativa para declividades negativas. Assim, temos que:

$\mathrm{MR}=\mathrm{M}-\mathrm{C}$, onde

$\mathrm{M}=1,5+2(\mathrm{~W}+\mathrm{L})(\mathrm{L} / \mathrm{W})^{2}+\mathrm{N}(\mathrm{W}+\mathrm{L})\left(1,5 \mathrm{~V}^{2}+\right.$

$0.35 \mathrm{VG})$

$C=n[(g(w+1) v / 3.5-((w+1)(g+6) 2 / W)+(25-V 2)]$

(MR) é a taxa metabólica em watts, (W) é o peso do indivíduo em quilogramas, (L) a carga 
extra, $(\mathrm{V})$ velocidade em metros por segundo, $(\mathrm{G})$ declividade em porcentagem e $(\mathrm{N})$ o fator do terreno.

A fim de reduzir o efeito da dependência da direção do movimento sobre a velocidade de deslocamento, Hare (2004) utilizou a fórmula de Tobler e converteu a velocidade em gasto calórico por km. Para isso utilizou a fórmula:

\section{$\mathrm{E}=48+30 / \mathrm{V}$}

onde (E) representa o gasto calórico dado em quilocaloria por quilômetro e $(\mathrm{V})$ é a velocidade expressa em $\mathrm{km} / \mathrm{h}$. A idéia do autor é que conforme aumenta o ritmo do caminhamento, maior é a eficácia do deslocamento até que a velocidade limite seja alcançada.

Um modelo alternativo foi proposto por McDonald (1961) e adaptado por Van Leusen (2000). O modelo propõe cálculos diferenciados para cada faixa fixa de declividade. Segundo o modelo teremos:

para faixas de $-40^{\circ}$ a $-20^{\circ}$ de declividade: $\mathrm{F} 1=0,000049 \mathrm{~V}^{2}-0,00415 \mathrm{~V}-0,13276 \mathrm{G}-$ $0,004692 \mathrm{G}^{2}-0,00005213 \mathrm{G}^{3}-0,000325 \mathrm{VG}$ $+0,000002036 \mathrm{~V}^{2} \mathrm{G}-0,8588$.

para faixas de $-20^{\circ}$ a $+5^{\circ}$ de declividade: $\mathrm{F} 2=0,00202 \mathrm{~V}+0,000021 \mathrm{~V}^{2}+0,0256 \mathrm{G}+$ $0,00154 \mathrm{G}^{2}+0,000044 \mathrm{VG}-0,00000314 \mathrm{~V}^{2} \mathrm{G}$ $+0,3515$.

e para as faixas de $+5^{\circ}$ a declividade máxima: $\mathrm{F} 3=\mathrm{V}^{*}(0,00275+0,049 \operatorname{sen}(\mathrm{S})) * \cos (\mathrm{S})+$ $\mathrm{V}^{2} *(0,00002-0,00033 \operatorname{sen}(\mathrm{S})) *(\cos (\mathrm{s}))^{2}+$ $0,396+0,17 \operatorname{sen}(S)$.

As superfícies de fricção criadas pelas funções F1, F2 e F3 são então somadas e o resultado é uma superfície final que apresenta o custo de deslocamento em termos de quilocaloria por $\mathrm{km}$.

Todos estes estudos tentam recriar sobre uma base fisiológica os padrões de locomoção e deslocamento humano dentro da paisagem. Definindo como um ser humano se desloca e quais as características associadas a esse deslocamento, é possível delinear zonas de exploração territorial, ou site catchment, de maneira mais realista, pois podemos avaliar quais os fatores que determinam do ponto de vista fisiológico o custo benefício do caminhamento.

\section{Reinterpretando a paisagem. Um modelo de site catchment}

A fim de testar a validade das novas metodologias de determinação de site catchment, utilizamos algumas das fórmulas acima em uma plataforma de GIS adotando como exemplo alguns sítios escolhidos de maneira direcionada a partir do Banco de Dados Tapirus. ${ }^{2} \mathrm{O}$ objetivo era selecionar pelo menos dois agrupamentos de sítios dentro e no entorno imediato da chamada Área Arqueológica de Serranópolis (Mapa 1). Esta região foi escolhida por apresentar uma indústria lítica bem distintiva. Esta área foi ocupada há 12.000 AP, por grupos caçadores-coletores, que produziam utensílios de pedra lascada, com estilo tecnológico próprio, tendo como "fóssil-guia", lesmas ogivais, pertencentes à Tradição Itaparica, fase Paranaíba (Teixeira 2007).

Os sítios arqueológicos escolhidos para análise foram os sítios Cachoeira 1, Cachoeira 3, Casa de Pedra, Aporé 1, Aporé 2, Aporé 3 e Lobe, por apresentarem em suas coleções vestígios associados à Tradição Itaparica em sua Fase Paranaíba.

Os sítios Casa de Pedra, Cachoeira 1 e Cachoeira 3 formam um complexo de oficinas líticas, localizado no vale do Rio Corrente, sudoeste do estado de Goiás. Esses sítios foram descobertos e pesquisados durante os

(2) O Banco de Dados Tapirus é um projeto que vem sendo executado pelos pesquisadores Dr. José Roberto Pellini, Me. Jonas Israel de Souza e Márcio Antônio Telles, em parceria com a 14. ${ }^{\text {a }}$ Regional do IPHAN (Instituto do Patrimônio Histórico e Artístico Nacional). Tal projeto visa cadastrar todos os sítios já identificados na área sob a responsabilidade da 14. ${ }^{a}$ Regional a fim de possibilitar não somente o estudo mais aprofundado da arqueologia do Centro-Oeste brasileiro, como também permitir políticas de manejo e preservação deste patrimônio. 


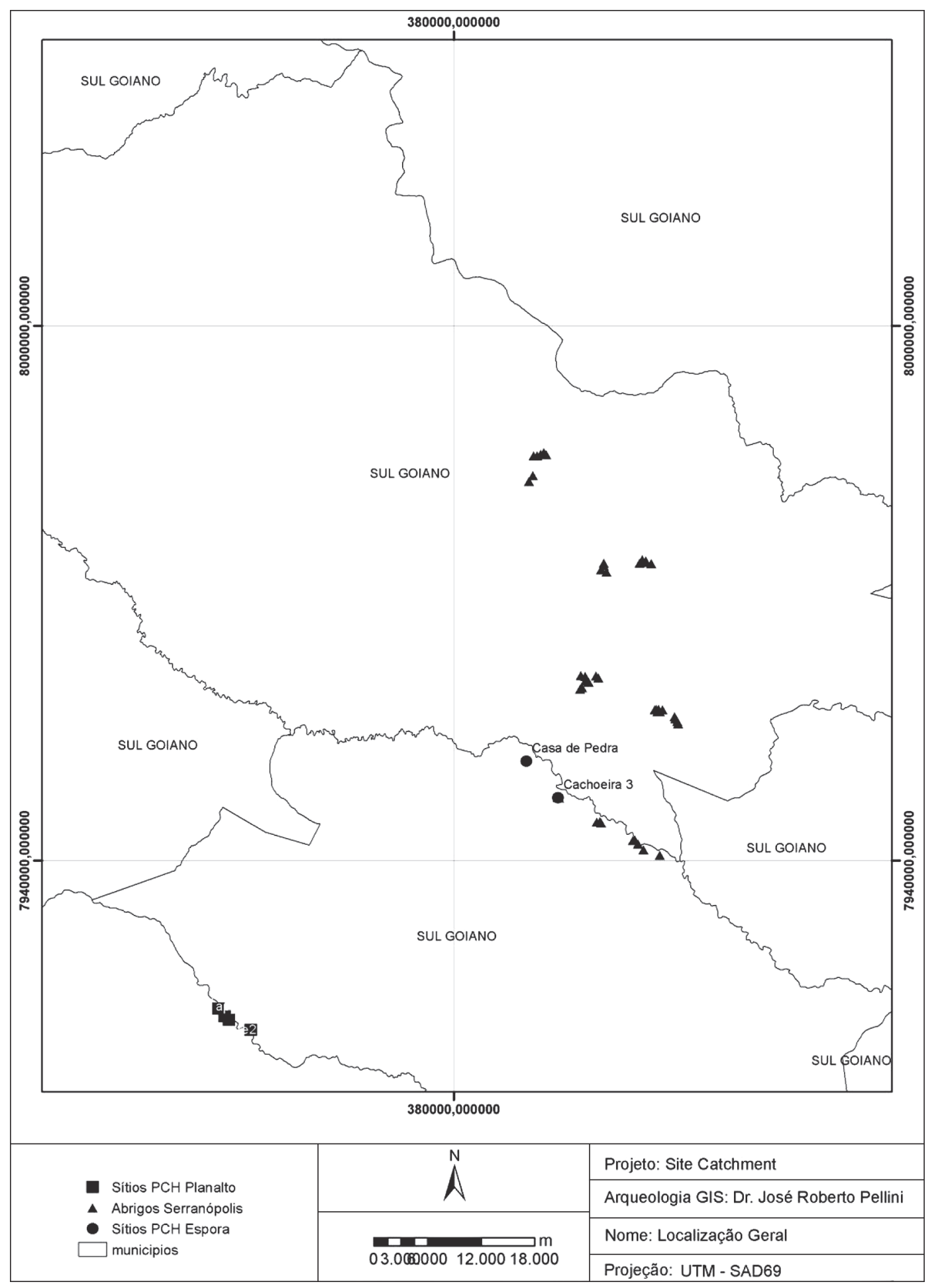

Mapa 1. Localização geral. 
trabalhos realizados ao longo do Projeto de Resgate do Patrimônio Arqueológico, Histórico e Cultural da UHE Espora no ano de 2006 (Teixeira 2007; Telles 2006).

Já os sítios Aporé 1, Aporé 2, Aporé 3 e Lobe, são pequenos acampamentos temporários que apresentam vestígios arqueológicos também na forma de material lítico lascado, mas distribuídos de forma dispersa e com baixa densidade (Pellini e Telles 2007). Estes sítios foram escavados dentro do escopo do Projeto Arqueológico da PCH Planalto. ${ }^{3}$

Para cada agrupamento foi definida uma área, um centro de gravidade, a fim de representar o ponto inicial de locomoção e deslocamento.

Com a área de pesquisa definida e os sítios selecionados, o passo seguinte foi obter um Modelo de Elevação Digital ${ }^{4}$ da área a fim de representar as elevações do terreno. Nosso Modelo de Elevação, ou simplesmente DEM, foi obtido junto a Shuttle Radar Topography Mission da NASA e conta com uma resolução de 30 x 30 m (Mapa 2). A partir da DEM, foi gerada uma superfície de declividade convertendo os valores de elevação em declividade expressas em graus, ${ }^{5}$ tarefa relativamente fácil com o módulo Spatial Analyst da suíte ArcGis 9.2.

A etapa final foi transformar os dados de declividade em tempo de deslocamento. $\mathrm{O}$ objetivo era obter uma superfície que representasse o tempo de caminhada baseado em velocidade, declividade e gasto calórico. Como a direção do deslocamento era um fator a ser considerado na análise, já que como dissemos acima o custo de locomoção pode variar de acordo com a direção do deslocamento,

(3) Tanto o Projeto da UHE Espora quanto o Projeto da $\mathrm{PCH}$ Planalto foram executados pela empresa Griphus Consultoria como parte dos processos associados ao Licenciamento Ambiental.

(4) Um Modelo de Elevação Digital é uma representação da topografia e da elevação de um terreno em forma de raster, ou imagem georreferenciada.

(5) Balstrom (2002) demonstrou através de dados de campo que declividades acima de $35^{\circ}$ positivo e negativo são pouco usuais e quase impraticáveis. Segundo o autor, o tempo médio requerido para se cruzar $1 \mathrm{~m}$ a uma declividade de $30^{\circ}$ é de 8 segundos. utilizamos o módulo PathDistance da mesma suíte ArcGis. Como fator de peso vertical foi utilizada a fórmula de Tobler, que foi modificada por Van Leusen (2000). O resultado final apresenta uma superfície que representa o tempo, em horas, gasto para percorrer uma dada distância a partir do centro do sítio (Mapas 3 e 4).

Com base nestes dados foram estabelecidos os catchments ou territórios de exploração para os dois agrupamentos de sítios. A proposta básica foi definir áreas dentro de um limite de 120 minutos de caminhamento como área principal de catchment. Sendo assim, os círculos concêntricos mostram a área que se pode percorrer dentro de tempo estimado em 120 minutos. Como podemos ver nos mapas 3 e 4, que definem as zonas de caminhamento em grupos de 2 horas, o primeiro círculo define os catchments imediatos aos centros de gravidade dos sítios. Cada contorno restante mostra as distâncias que se podem percorrer dentro de um período de 2 horas de maneira subseqüente.

Com as áreas de catchment definidas, podemos analisar quais as características ambientais que formam cada território de exploração, ou seja, quais os recursos e características que estão a 120 minutos do sítio. Utilizando dados fornecidos pela Seplan (Secretaria de Planejamento) do estado de Goiás, podemos caracterizar a zona de 120 minutos de catchment para os sítios da $\mathrm{PCH}$ Planalto como constituída por latossolos (52\%), plintossolos (22\%) e neossolos (26\%). Os arenitos cobrem cerca de $87 \%$ da área e os quartzos, 11\%. A vegetação atual é representada por pasto $(63 \%)$, cerrado $(23 \%)$ e mata (14\%). Já no caso do catchment associado aos sítios da UHE Espora, arenitos cobrem cerca de $35 \%$, basaltos $22 \%$ e areias e argilas $43 \%$. Os latossolos representam o tipo de solo predominante, com $57 \%$, seguidos dos neossolos, com 43\%. Quanto à vegetação atual, temos na área do catchment $71 \%$ de pasto, $12 \%$ de cerrado e $17 \%$ de mata, principalmente na forma de mata ciliar. Esta rápida configuração mostra que a área de catchment dos sítios da UHE Espora, pelo 


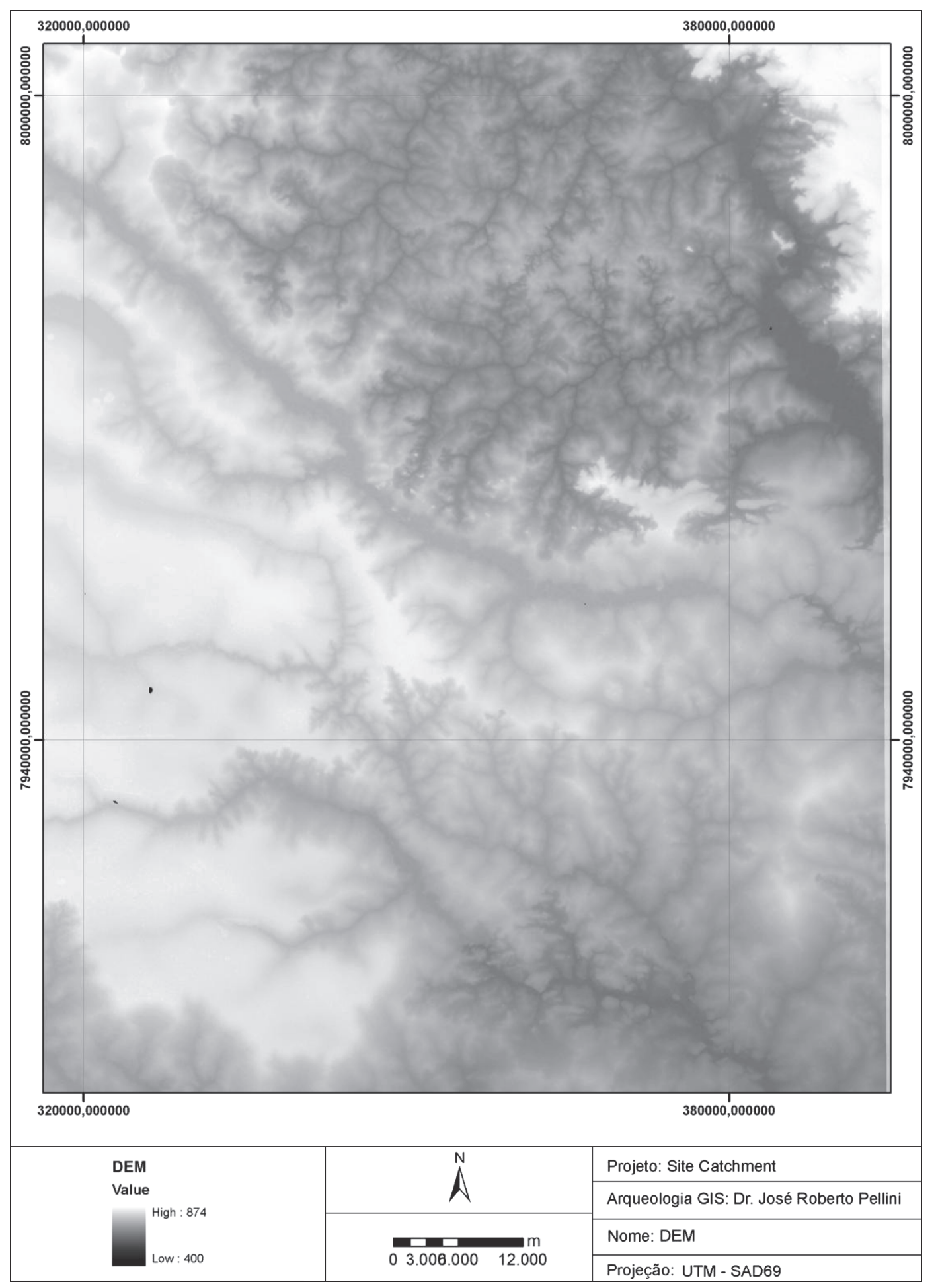

Mapa 2. Modelo de elevação digital - Área de estudo. 


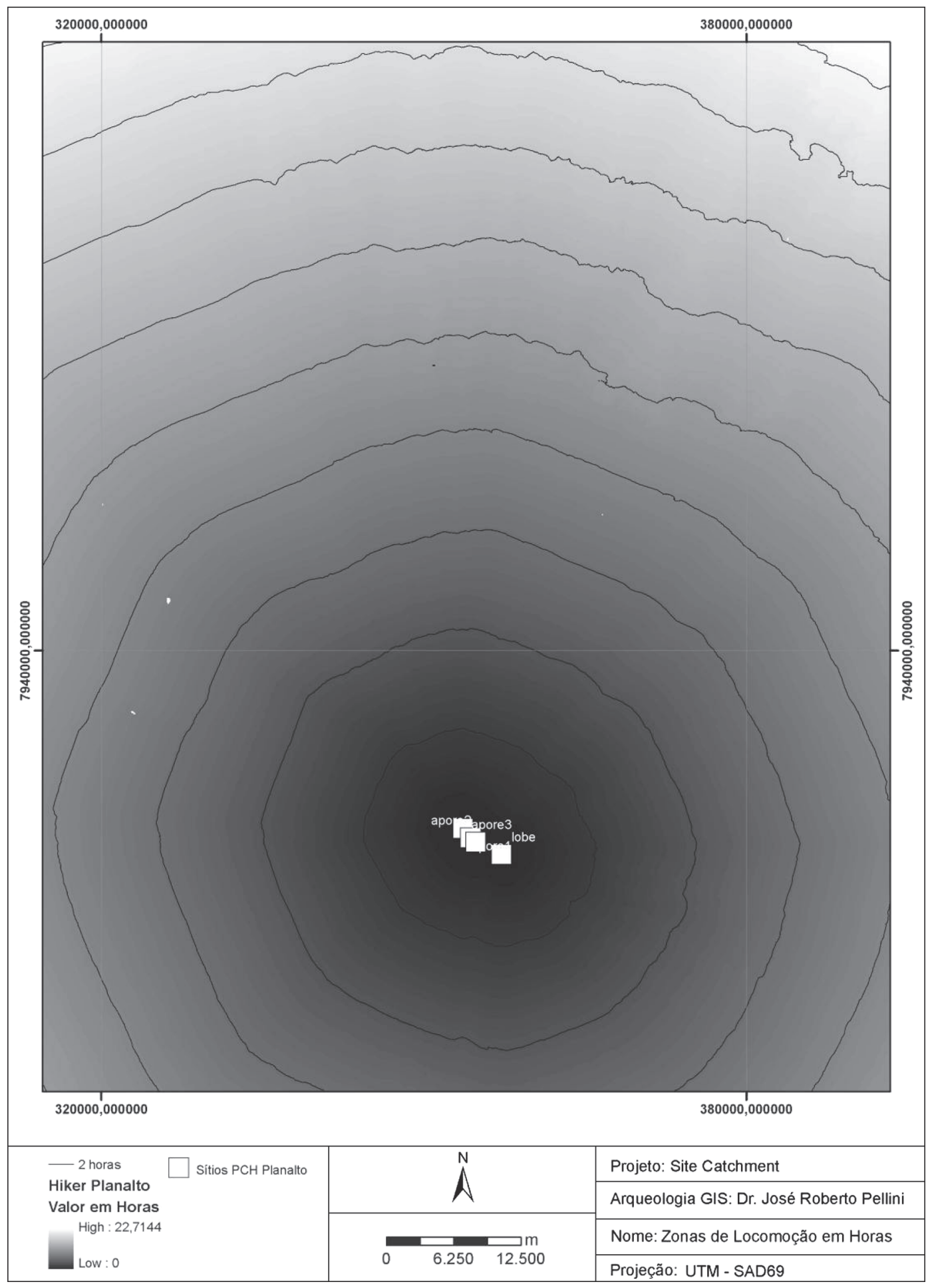

Mapa 3. Zonas de locomoção - Sítios arqueológicos PCH Planalto. 


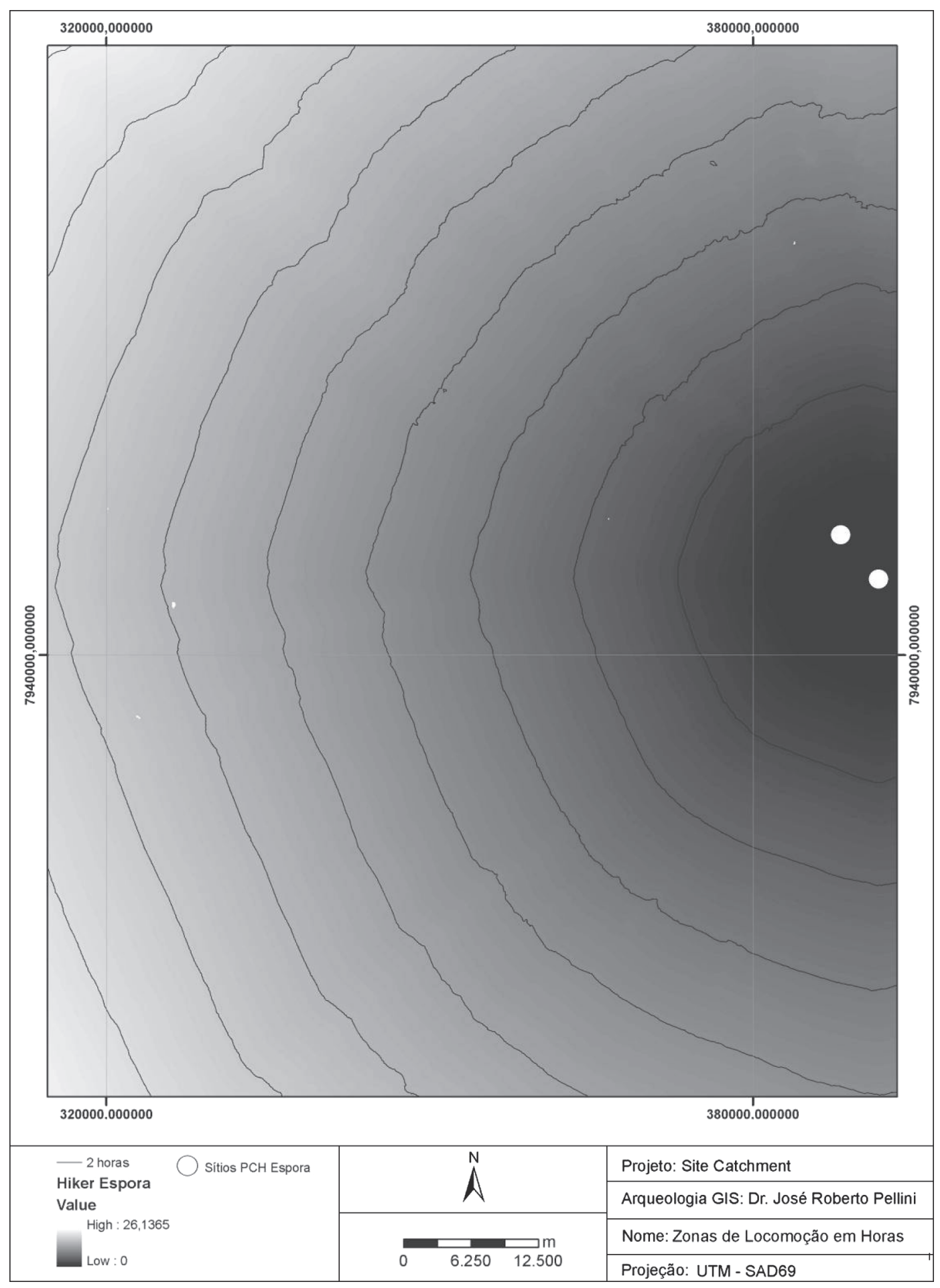

Mapa 4. Zonas de locomoção - Sítios arqueológicos PCH Espora. 
menos do ponto de vista da obtenção de matéria-prima, é mais diversificada. Quanto à qualidade dos solos para plantio, a área de exploração territorial da UHE Espora mostra uma maior capacidade agrícola, pois é formada por $72 \%$ de solos eutróficos, enquanto a área de catchment dos sítios da PCH Planalto apresentam apenas 34\% de solos deste tipo, prevalecendo assim os solos distróficos de baixa qualidade. ${ }^{6}$ A baixa qualidade do solo associada à baixa diversificação dos recursos explica a não existência de sítios mais permanentes na área da PCH Planalto. A própria não existência de fontes de argila dentro da zona de 120 minutos é em si um empecilho para o desenvolvimento de sociedades ceramistas e horticultoras nesta área. Em contrapartida, a maior diversidade dos catchments da área de Serranópolis é evidenciada diretamente na estratigrafia dos abrigos, onde se observa não somente a existência de uma maior quantidade de matérias-primas líticas, mas também a presença de cerâmica e restos faunísticos variados, o que não acontece nos sítios da PCH Planalto.

Inserindo nas análises os Abrigos de Serranópolis (Schmitz 1997), podemos observar que os sítios da UHE Espora se inserem no limite da principal zona de catchment dos Abrigos, ou seja, mesmo estando na periferia do território de exploração, inserem-se dentro da área de domínio dos Abrigos (Mapa 5). Ao mesmo tempo podemos ver que os sítios da PCH Planalto, mesmo apresentando uma cultura material semelhante, inserem-se completamente fora do catchment de Serranópolis.
Com as superfícies de deslocamento criadas e os territórios de exploração analisados, o passo final é estabelecer quais seriam as rotas mais eficientes ligando os sítios da UHE Espora, os sítios Abrigos de Serranópolis e os sítios da PCH Planalto. Utilizando como base de fricção as superfícies de deslocamento criadas com as fórmulas de Tobler, foram definidos quais os melhores acessos interligando as áreas em estudo. Como podemos ver no Mapa 6, se traçarmos uma linha reta (a menor distância linear do ponto de vista euclidiano) interligando os pontos escolhidos para análise e compararmos com as distâncias de maior eficiência, veremos que as distâncias que ligam os sítios da PCH Planalto com o agrupamento de sítios da UHE Espora são de 43,314 km no sistema euclidiano contra 44,405 km da rota de eficiência. Interligando os sítios da UHE com o centro do agrupamento dos Abrigos de Serranópolis teremos 18,662 contra $18,117 \mathrm{~km}$. E por fim a interligação dos sítios da PCH Planalto com os Abrigos de Serranópolis apresenta 59,653 km no sistema linear e 58,200 km no sistema de maior eficiência. Analisando mais detalhadamente os valores obtidos veremos que, excetuando a relação Planalto/Abrigos, todas as demais rotas de eficiência se apresentaram maiores que a rota em linha reta. Isto se justifica pelo fato de que nem sempre a menor distância entre dois pontos é a melhor rota dentro da paisagem. Em nosso caso específico, embora as rotas de maior eficiência sejam maiores em termos de quilômetros, elas são as rotas em que o gasto calórico é menor e em que a velocidade de deslocamento é maior, ou seja, em que o tempo de deslocamento é menor (Tabela 1).

Tabela 1

\begin{tabular}{lccc}
\hline \multicolumn{4}{c}{ Comparação das distâncias em km } \\
\hline & Planalto/Espora & Espora/Abrigos & Planalto/Abrigos \\
\hline \hline Rota linear & 43,314 & 18,662 & 59,653 \\
Rota de eficiência & 44,405 & 18,117 & 18,117 \\
\hline
\end{tabular}

(6) As análises paleo-ambientais que estão sendo conduzidas na região relativas ao Projeto de Levantamento e Resgate da UHE Espora irão fornecer maiores subsídios para a análise das áreas de catchment de ambos os agrupamentos. 


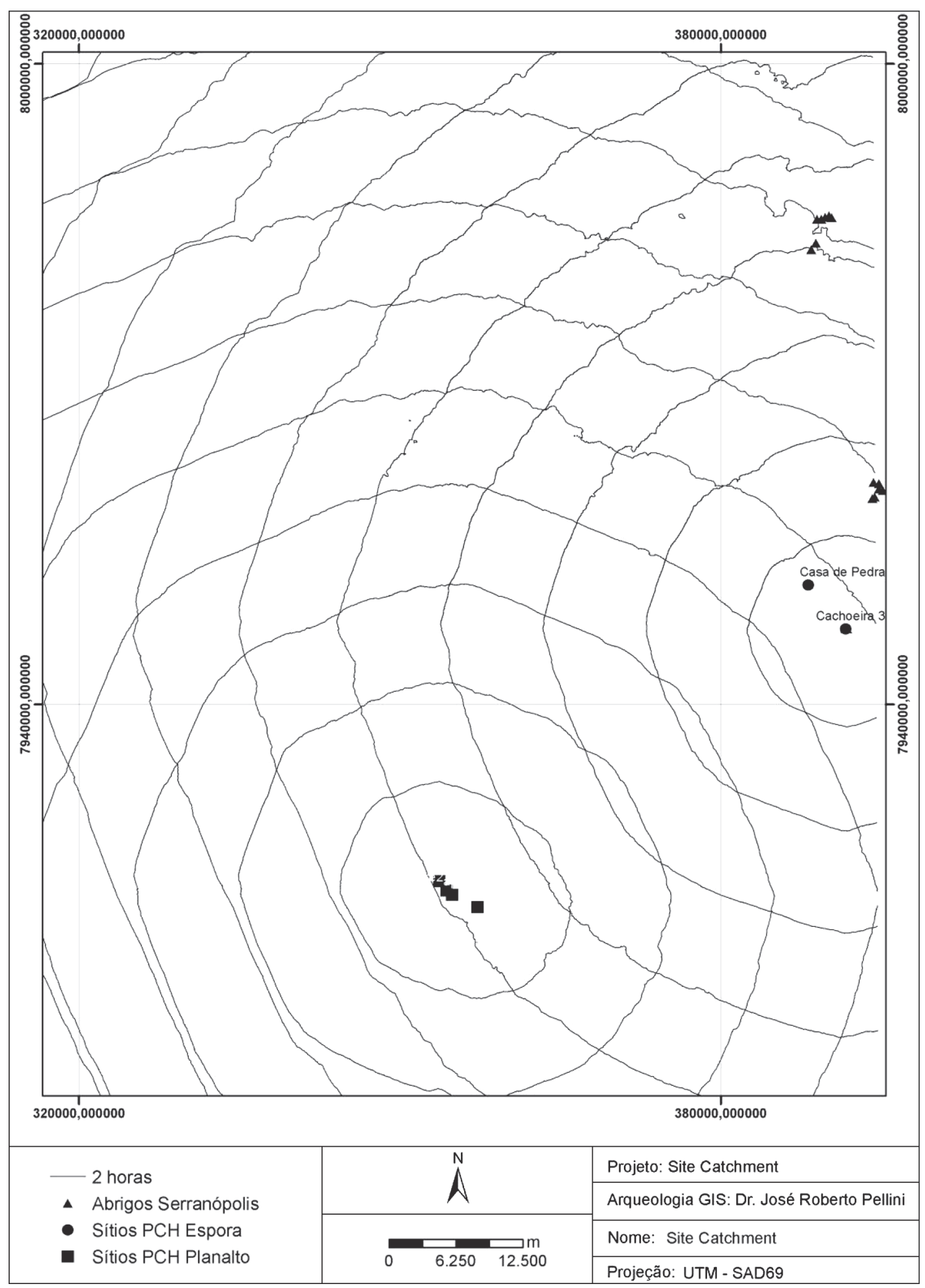

Mapa 5. Zonas de sobreposição. 


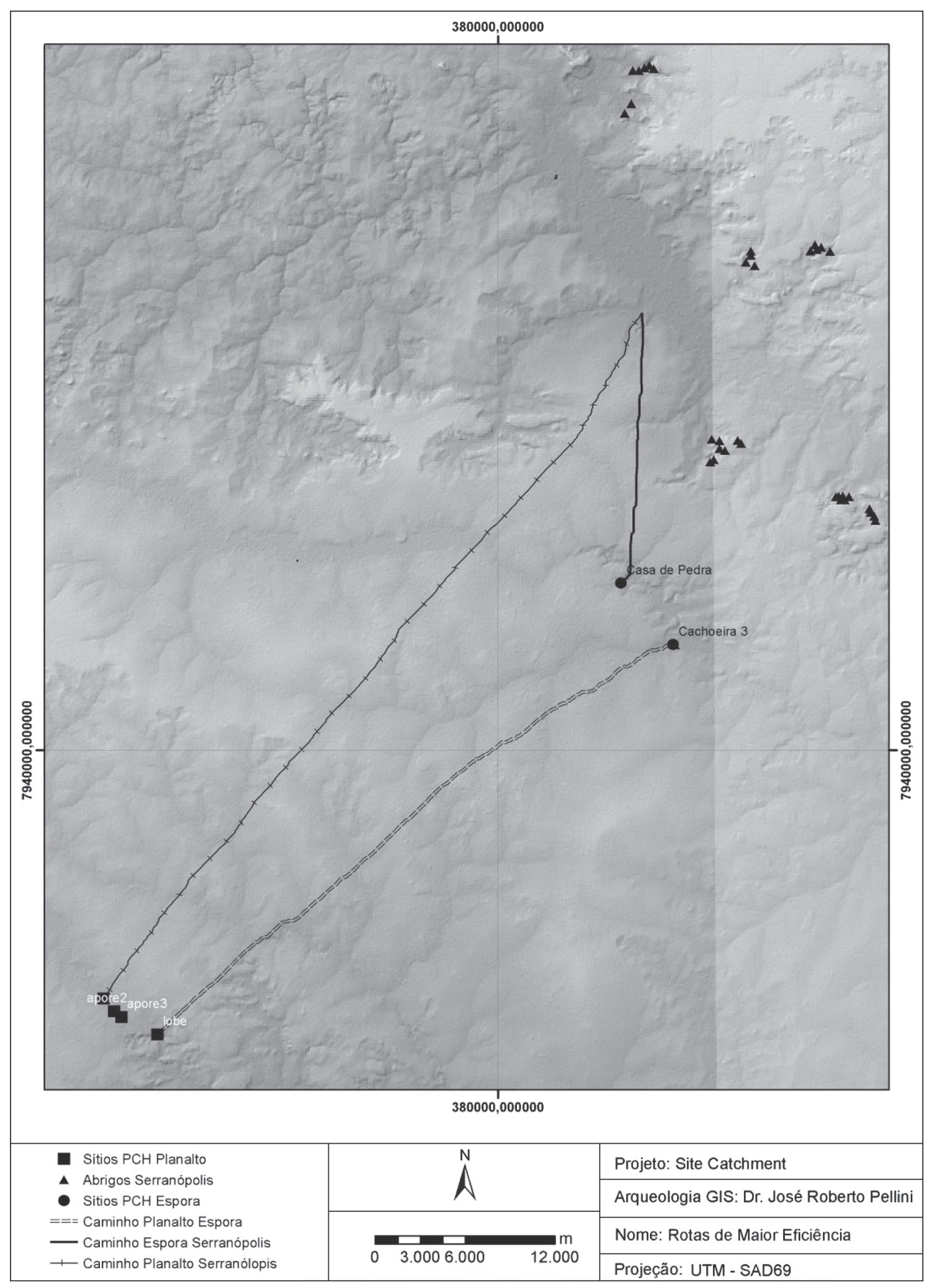

Mapa 6. Rotas de deslocamento. 


\section{Conclusão}

Os sistemas de deslocamento e locomoção têm um peso importante na maneira como os indivíduos concebem o meio que os cerca. Compreender este sistema é fator essencial na tentativa de entender como os grupos exploravam, assimilavam e compreendiam o mundo a sua volta. Distância, locomoção e acessibilidade são aspectos culturais e assim devem ser encarados nas análises de site catchment. Não basta traçar um círculo ao redor do sítio com um determinado comprimento de raio, essa é uma abstração cartesiana que pouco tem a ver com os intrincados processos de deslocamento. Faz-se necessário definir catchment com base em dados que remetam ao indivíduo ou ao grupo. Faz-se necessário conceber distância em termos humanos e não geográficos. Nessa linha, a determinação de catchments baseada em medidas fisiológicas de custo calórico e velocidade de transposição de terreno são mais adequadas para a determinação de territórios de exploração, pois permitem a comparação do custo-benefício das atividades desenvolvidas dentro do catchment.

O principal objetivo deste artigo foi apresentar um modelo de site catchment mais realístico e demonstrar o que a nosso ver são as vantagens intrínsecas no processo de análise. $\mathrm{O}$ passo a seguir é inserir nas análises fatores de impedimento, ou seja, barreiras físicas e culturais que limitam o deslocamento, bem como trabalhar com superfícies de fricção que sejam cada vez mais próximas da realidade humana.

PELLINI, J.R. A Physiology of Landscape: Travel, GIS and Sites Catchment. A New Approach. Revista do Museu de Arqueologia e Etnologia, São Paulo, 17: 000-000, 2007.

Abstract: Locomotion and distance are essential elements of the human life. However, in spite of its importance, these concepts have been treated in a simple way for archaeology, mainly for Site Catchment Analysis. The most traditional models of Site Catchment deal with the space as a neutral cartesian element. In the last years the use of the GIS has allowed the determination of Site Catchments in more realistic way, mainly when considering physiological factors and elements of perception in the modeling of the exploration territories. The proposal of this article is to present an example of the use of these new models and to test its validity as interpretation method.

Keywords: Site Catchment - Travel - GIS.

\section{Referências bibliográficas}

BALSTROM, T.

2002 On Identifying the Most Time Saving Walking Route in a Trackless Mountainous

Terrain. Danish Journal of Geography, 102: 51-58.

BECK, C.; TAYLOR, A.

2003 Rocks are Heavy. Transport Costs and Paleoarchaic Quarry Bahavior in the Great Basin. Journal of Anthropological Archaeology, 21 (4): 481-507.
DUGGAN, A.; HAISMAN, M.

1992 Prediction of the New Metabolic Cost of Wlaking with and without Loads. Ergonometrics, 35: 417-420.

GAFFNEY, V.; STANCIC, Z.

1991 GIS Approaches to Regional Analysis. A Case Study of the Island of Hvar. Ljubljana. University of Ljubljana.

HAGERSTRAND, T.

1962 Geographical Measurements of Migration. 
In: Sutter, J. (Ed.) Les Desplacements Humains. Aspects Methodologiques de Leur Mesure. Entretiens de Monaco en Sciences Humaines, 24-29 Mai, 1962: 31-62.

HARE, T.

2004 Using Measures of Cost Distance in the Estimation of Polity Boundaries in the Postclassic Yautepec Valley, Mexico. Journal of Archaeological Science, 31: 799-814.

IMHOLF, E.

1950 Gelaende und Karte. Zurich: Rentsch.

LIMP, W.

1990 Intersite Analysis: Aboriginal Use of the Rush Locality. In: Sabo, G. (Ed.) Archaeological Investigation at 3MR80 Area $D$ in the Rush Development Area. Buffalo National River. Professional Paper 38, vol 1: 295-345.

LLOBERA, M.

1996 Exploring the Topography of Mind: GIS, Social Space and Archaeology. Antiquity, 70: 612-622.

2000 Understand Movement: A Pilot Model Towards the Sociology of Movement. In: Lock, G. (Ed.) Beyond the Map: Archaeology and Spacial Technologies. Amsterdam, IOS Press: 65-84.

MARBLE, D.

1996 The Human Effort Involved in Movement over Natural Terrain: A Working Bibliography. Report of Department of Geography, Ohio State University.

MCDONALD, I.

1961 Statistical Studies of Rocorded Energy Expediture of Man". Nutritional Abstracts and Reviews, 31 (3): 739-762.

MORRILL, R.; PITTS, F.

1967 Marriage, Migration and the Mean Information Field: A Study in Uniqueness and Generality. Annals of the Americam Association of Geographers, 57 (2): 401-422

PANDOLF, K.; GIVONI, B.; GOLDMAN, R.

1977 Predicting Energy Expediture with Loadas While Standing or Walking Very Slowly. Journal of Applied Physiology, 43: 577-581.

PELLINI, J.R.; TELLES, M.

2007 Relatório Final do Projeto de Resgate Arqueológico da PCH Planalto. São Paulo.

SCHMITZ, P.I.

1997 Arqueologia nos Cerrados do Brasil Central.
Serranópolis II. São Leopoldo, UNISINOS, Instituto Anchietano.

TEIXEIRA, E.

2007 Variabilidade Tecnológica nos Sítios Cachoeira 1, Cachoeira 3 e Casa de Pedra, Sudoeste de Goiás. Comunicação apresentada no XIV Congresso da Sociedade Brasileira de Arqueologia, Florianópolis, 30 de setembro a 5 de outubro de 2007.

TELLES, M.

2006 Projeto de Resgate Arqueológico, Histórico e Cultural da UHE Espora. Goiânia.

TOBLER, W.

1993 Non-isotropic Geographic Modeling. Three Presentation on Geographical Analysis and Modeling. National Center for Geographic Information and Analysis. Techical Report, 93 (1).

VAN LEUSEN, M.

2000 Exploring Digital Archaeological Landscape: A Discussion of Regional Map Forming Patterns and Processes. University of Gronningen, PhD Thesis.

VERHAGEN, P.; MCGLADE, J.; GILI, S.; RISCH, R.

1995 Some Criteria for Modelling SocioEconomic Activities in the Bronze Age of South East Spain. Lock, G.; Stancic, Z. (Eds.) Archaeology and Geographical Information Systems: 187-209.

VERHAGEN, P.; GILI, S.; MICO, R.; RISCH, R.

1999 Modelling Prehistoric Land Use Distribution in the Rio Águas Valley (SE Spain). DingWall, L. (Ed.) Archaeology in the Age of Internet. Proceedings of the CAA 97 Conference. BAR International Series, 750.

VITA FINZI, C.; HIGGS, E.

1970 Prehistoric Economy in the Mount Carmel Área of Palestine. Site Catchment Analysis. Proceedings of Prehistoric Analysis, 36: 1-37.

WHITLEY, T.; HICKS, L.

2003 A Geographic Information System Approach to Understand Potential Prehistoric and Historic Travel Corridors. Southeastern Archaeology, 22 (1): 77-91,

WOOD, B.; WOOD, Z.

2006 Energetically Optimal Travel Across Terrain: Visualizations and a New Metric of Geographic Distance with Archaeological Applications. Proceedings of SPIE, Electronic Imaging. January, San Jose: 1-7. 